\title{
LA-6767-MS
}

Informal Report

UC.20

Issued: April 1977

\section{Effects of Flux Conservation on the Field Configuration in Scyllac}

by

P. C. T. Van der Laan

of the University of California LOS ALAMOS. NEW MEXICO 87545

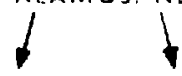

An Alfirmative Action/Equal Op; ortunty Employer 
This work was supperted by the US Energy Research and Development Administraticn, Division of Magn-tic Fusion Energy, Applied Plasma Branch.

\footnotetext{
Printed in the Linited States of America. Available from National Technical Inforination Service

U.S. Department of Commerce

5285 Port Royal Road

Springficld, VA 22161

Price: Printed Copy \$3.50 Microfiche $\$ 3.00$

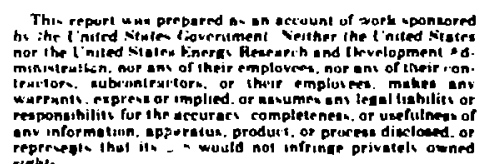

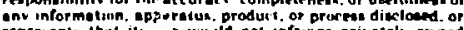

rifente
} 
EFFECTS G FLUX CONSERVATION ON THE FIELD CONFIGURATION IN SCYLLAC

by

P. C. T. Van der Laan

\begin{abstract}
Flux conservation in Scyllac-type experiments shows up in two ways. First of all the poloidal flux between the outside edge of the fiasma and the inside of the coil is conserved. This requires a net longitudinal current in the plasma, to cancel the poloidal flux caused by the helical stellarator fields. An expression for this net current is derived, and effects that could occur in sector experiments are discussed.

The flux conservation inside the conducting plasma leads to a conservation of the local rotational transform. Since the pinch itself is surrounded by a well-conducting low-density plasma, the rotational transform is conserved in a wide region. Depending on tile time history of the applied fields, volune currents are induced in this region, as is shown for two examples. Although an additional capacitor bank can be used to cancel the net current, a cancellation of all the volume currents is extremely difficult. The resulting equilibrium configurations differ considerably from the Scyllas equilibria without volume currents, which are used in stability calculations.
\end{abstract}

\title{
I . INTRODUCTION
}

In calculations on Scyllac, various assumptions are made to arrive at a model for the equilibrium configuration. One of these assumptions is about the pressure Frofile; sharp skin or diffuse models have been used. Other assumptions have to be made about the magnetic field configuration in and near the plasma, before the field profiles can be matched to their boundary values.

In an often used expansion, the zeroth order is a theta pinch and all firstorder quantities are witten as a product $f(r) \cos (\theta+h z)$ where $h$ is equal to $2 \pi$ divided by the length of one helical period. The integral $\oint B_{\theta} d 1$ on any cylinder $r=$ constant is therefore zero, since botin the zero order theta pinch and 
the first order $B_{\theta}$ field do not contribute to the integral. This means that the total = current on any cylinder is zero. This represents a specific shocio fur a class of equilibria. Cther choices are passible such as the one made by Weitzner, ${ }^{l}$ who describes a calculation where a net z current flows cn one surface, namely, the surface of the sharp skin plasma.

In the real experiment, processes during the formation phase determine what type of equilibrium the plasma will approach. This is obvirus for the pressure profile, which is completely determined by the implosion process. The field profiles will likewise be determined by the plasma motion and the field diffusion which take place during the formation phase. Predictions about the resulting field profiles are difficult to make when the formation process is slow and when much turbulence is being excited as in Tokamaks and in turbulent heating experiments. When powerful implosion heating is being used, not much interdiffusion of field and plasma can occur. Plasma and field are then frozen together, which means that fluxes are conserved. If fluxes are conserved in a detailed sense, in other words, between adjacent magnetic surfaces, the rotational transform (or the $q$ value) on any magnetic surface stays constant. This behavior has been observed in screw pinches for many years ${ }^{2,3}$ and is expected to become important in Tokamaks with powerful auxiliary heating (flux-conserving Tokamaks). ${ }^{4}$ In Scyllac, as in all powerful theta pinches, the implosion heating is certainly strong and fast enough to ensure the validity of this conservation model.

In this report we study the question whether the equilibria chosen in the theoretical papers are compatible with the model in which fluxes and rotational transforms are being conserved. This is an especially important question if the presence of a low-density plasma outside the central column is taken into account. This outside plasma has a low pressure and does not carry appreciable perpendicular currents, but it can carry large force-free currents. When the outside plasma is included the region in which flux conservation takes place is considerably widened.

Flux conservation sholild first of all be considered in a giobal sense, for the nonconducting region between the outer edge of the plasma and the external circuit, which can be the inside of the coils. Since the total flux enclosed should stay constant, the plasma carries a net current which cancels any changes in the enclosed flux (Sec. III). The difference with earlier studies by Sugisaki, ${ }^{5}$ Miller, ${ }^{6}$ and in Gerching ${ }^{7}$ is that the outer edge of the conducting 
medium is now assumed to coincide with the quart: wall. This simplifies the calculation of the net current but does not answer the question where this current is flowing.

To find the current distribution, one should zonsider detailed flux conservation, or rather the conservation of rotational transform on each magnetic surface. A general description along those lines is d.fficult and we give therefore two simplified examples in Sec. IV. The first one describes what internal $z$ currents one can expect when an $\ell=1$ field is applied with the same time history as the mair $B_{z}$ field. The second example describes the internal current distribution which results when an $\ell=2$ field is appi ied to an already formed theta pinch.

\section{ROTATIONAL TRANSFORM AND POLOIDAL FLUXES}

The rotational transform generated inside a Scyllac coil results from the $\ell=1$ and $\ell=2$ fields. The combination of a helical field and the main $B_{2}$ field distorts the field lines into spirals with one gyration per period of the coil and a much slower rotation around the axis of the coil. This slow rotation can be expressed in terms of a rotational transform which, for the full torus and for a vacuum field is given by 8

for $\ell=1 \quad l=\frac{\pi h R A_{1}^{2}}{B_{z}^{2}}\left\{1+0\left(h^{2} r^{2}\right)\right\}$

and

for $i=2 \quad l=\frac{2 \pi R A^{2}}{4 s_{z}^{2}}\left\{1+0\left(h^{2} r^{2}\right)\right\}$

where $h$ equals $2 \pi$ divided by the length of one helical period, $R$ is the major radius of the torus, and $A_{1}$ and $A_{2}$ are coefficients characterizing the amplitudes of the helical field. In the limit of small hr, $A_{l}$ equals the absolute value of the $\ell=1$ field whereas $A_{2}$ equals the absolute value of the $\ell=2$ field at a given radius, divided by that radius. The number of helical periods on the circumfererice of the torus is hR.

The presence of the rotational tionsform implies that a poloidal flux $\phi_{\ell}$ is generited. The equation relating the toroidal flux and the poloidal flux is 9 


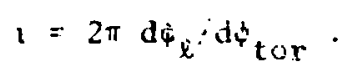

Outside the dense cenc: ${ }_{1} 1$ plasma $d \phi$ tor can be written as $2 \pi r B_{z} d r$, so that the poloidal flux follows froa

$$
\phi_{\ell}=\int l_{\ell} B_{z} r d r
$$

where the flux is that between the two radii used as the limits for the integration at the right hand side.

\section{NET TOROIDAL CIJRRENT}

\section{A. Simple Case}

The flux between the inside radius $b_{c}$ of the coil and the outer edge of the conducting plasma at $r=b_{p}$ can be calculated from Eq. (4).

$$
\phi_{\ell}=1 / 2 B_{z}\left(b_{c}^{2}-b_{p}^{2}\right) \iota_{\ell}
$$

where the terms of order $h^{2} r^{2}$ in Eqs. (1) and (2) have been neglected. Also neglected is the influence of the shape of the plasma surface on the fields. This is reasonable when the radius of the dense plasma $a$ is much less than the outer radius of the low-density plasma $b_{p}$ [compare Eq. (12)]

The toroidal plasma encloses the flux $\phi_{\ell}$ which rises with $B_{z}$. This induces a toroical plasma current $I_{z}$, whose flux $\phi_{c}$ should be equal and opposite to $\Phi_{\ell}$. In case the coil is continuous along the torus the ratio of $\phi_{c}$ to $I_{z}$ is given by the inductance for a coaxial cylinder of length $2 \pi R, \mu_{o} R \ln b_{c} / b_{p}$. The current $I_{z}$ is then

$$
I_{z}=\frac{B_{z}\left(b_{c}^{2}-b_{p}^{2}\right)}{2 \mu_{0} R \ln \left(b_{c} / b_{p}\right)} l_{\ell}
$$

The flux $\phi_{c}$ depends on the net current within the radius $b_{p}$. Equation (6) therefore gives this net current, but no information at all on how this current is distributed within the plasma. 


\section{B. Numerical Examples}

In the Scyllac full torus experiments of summer $i 974^{10}$ with $\ell=1$ coils, the toroidal current calculated from the Eqs. (1) and (6) for the parameters $\mathrm{R}=4 \mathrm{~m}, \mathrm{~b}_{\mathrm{c}}=7.1 \mathrm{~cm}, \mathrm{~b}_{\mathrm{p}}=4.4 \mathrm{~cm}, \mathrm{~B}_{z}=4 \mathrm{~T}, \mathrm{~h}=15 \mathrm{~m}^{-1}$, and $\mathrm{B}_{\ell=1} / \mathrm{B}_{2}=0.064$ turns out to be $1.99 \mathrm{kA}$, which is of the same order as the currents that were actually measured.

The $\ell=2$ field of the coils that were used in 1977 would induce a current, in a full torus, which calculated from Eqs. (2) and (6) turns out to be $15.4 \mathrm{kA}$, for the parameters $R=4 \mathrm{~m}, \mathrm{~b}_{\mathrm{c}}=10.6 \mathrm{~cm}, \mathrm{~b}_{\mathrm{p}}=4.4 \mathrm{~cm}, \mathrm{~B}_{\mathrm{z}}=2 \mathrm{~T}, \mathrm{~h}=6 \mathrm{~m}^{-1}$, and $A_{2}=2.64 \mathrm{~T} / \mathrm{m}$. The rotationai transform caused by the $\ell=1$ field, which in this case equals $0.265 \mathrm{~T}$, is smaller than that caused by the weaker $\ell=2$ field and consequently the current, which the $\ell=1$ field would induce in the torus, is only $2.8 \mathrm{kA}$.

\section{Special Cases, End Effects}

For the calculation of the net plasma current in Sec. II.A the theta-pinch coils were assumed to be continuous in the toroidal direction. When the various sections are connected with some additional inductance between the sections, the flux $\phi_{c}$ which has to cancel $\phi_{\ell}$ will be

$$
\phi_{c}=I_{z}\left\{\mu_{o} R \ln \left(b_{c} / b_{p}\right)+L_{e}\right\}
$$

where the total additional inductance is given by $L_{e}$. The Eqs. (5) and (7) will give an $I_{z}$ current smaller than that given by Eq. (6).

In the case of a complete break in the coil the inductance $L_{e}$ increases to the $z$ inductance of the toroidal coil which is

$$
L_{z}=\mu_{0} R|\ln (8 R / b)-2|
$$

where $b$ is the outside radiis of the coils and $R$ the major radius. With $R=4 \mathrm{~m}$ and $b=0.15 \mathrm{~m}, \mathrm{~L}_{z}=16.9 \mu \mathrm{H}$. This inductance has to be compared to the coaxial inductance $\mu_{o} R \ln \left(b_{c} / b_{p}\right)$ to see by what factor the $I_{z}$ found from Eq. (s) is reduced by a break.

The net $z$ current can be reduced if the coil has a break, or can be canceled by means of an auxiliary capacitor bank. Neither of those methods ensures however, that the $z$ currents will be zero throughout the plasma (Sec. IV). 
Many Scyllac experiments have been carried out in sectors. Since in those machines all magnetic fiold lines intersect the insulating wail at the ends, conservation of poloidal flux and conservation of rotational transform would seem not to occur, so that the conclusions of this report would not be relevant. There are.however, two effects ${ }^{11}$ that can cause conservation of poloidal flux as in the full torus.

1. A change in rotational transform in a sector experiment leads to angular velocities of the fieid lines and the low-density plasma near the ends. In a long machine this rotation can correspond to an appreciable kinetic snergy, ever for fairly low outside densities. A polarization current, flowing across the field lines is needed whenever the angular velocity is changing. Since this polarization current continues as a $z$ current on the axis and at the outer edge of the outside plasma, it tends to screen off the change of the rotational transform. This mechanism leads to a complete screening of $\dot{f}$, as in a full torus, for fast changes of the applied rotational transform and to easy penetration for slow changes. To be in the slow category the frequency $\omega$, with which the applied rotational transform changes, must satisfy 11,12

$$
\omega^{2} \ll 2 V_{A}^{2} / L^{2}=2 B^{2} / \mu_{o} \rho L^{2}
$$

where Alfuén velocity $V_{A}$ and the mass density $p$ have to be taken for the outside plasma and where $L$ is the length of the sector. For $B=4 T, L=8 \mathrm{~m}$, and for an outside deuterium plasma of density $10^{14} \mathrm{~cm}^{-3}$, the limiting $\omega$ turns out to be $1.1 \times 10^{6} \mathrm{~s}^{-1}$.

2. A change of rotational transform in a sector is accompanied by a change of poloidal flux. This change of flux causes radial E fields. Because all field lines leave the machine at the ends, a shorting of the $E$ fields along the inner surface of the insulating discharge tube may occur. A perfect shorting would lead to a conservation of rotational transform, as in a full torus.

Both effects mentioned here make it difficult to predict what role the outside plasma will play in a sector, and make the extrapolation from a sector to a full torus problematic. 


\section{INTERNA!. CURRENT DISTRIBUTION}

Tile precise distribution of the 2 currents in the plasma is more important than the net $z$ current itself, because even when the net current is carceled, remaining opposing $z$ currents in the plasma may still have their influence on equilibrium and stability.

A general descr ption of the internal current distribution is not easily given, because too many factors play a role. The time history of the externai fields, $B_{2}, B_{\ell}$, and $B_{\theta}$, the inward motion of fields and plasma during the formation, and the time dependence of the distortions of the central plasma all influence the $z$ currents.

To illustrate the problem we give two exampies; in the first one an $\ell=1$ helical field and the main field are applied with the same time history, as by a shaped coil, whereas in the second example, an $\ell=2$ helical field is applied late in time as by a feedback or a trimming field coil.

A. Helical $\ell=1$ Field Rising with Main Field

In this first example both $B_{\ell=1}$ and $B_{z}$ rise with the same time history, as in the case when tney are both produced by a shaped coil. All field lines come in from the outside and move inwards into the plasma, which extends to the radius $b_{p}$. When the field lines are at the position $b_{p}$ two effects contribute to their rotational transform; the helical field produces in ' $\ell$ given by Eq. (1) and the longitudinal plasma current produces a rotational transform ${ }^{l} c$, which is related to the current by

$$
l_{c}=\frac{\mu_{0} R I_{z}(r)}{r^{2} B_{z}}
$$

For a continuous toroidal coil, or tor perfect shorting at the ends of a sector, Eq. (6) gives the current. Substitution of this current and of $r=b_{p}$ into Eq. (10) yields ' $c$ ', which turns out to be opposite to and larger than ' $\ell$. The resulting rotational transform is then

$$
\mathrm{r}_{\text {res }}=\frac{2 \ln \left(b_{c} / b_{p}\right)-b_{c}^{2} / b_{p}^{2}+1}{2 \ln \left(b_{c} / b_{p}\right)} l_{\ell},
$$


where the coefficient of $l_{\ell}$ is negative and tis absolute value exceeds 1 for $b_{c} / b_{p}>1.87$.

Once the field lives have entered the conducting plasma, they conserve the rotational transform 'res. Figure la shows the resulting distribution of the rotational transforms, ${ }^{l} l,{ } c$, and 'res, and the $B_{f}$ field caused by the voiume currents, for the hypothetical case of a low-beta central plasme. The current density $j_{z}$ is homogeneous for this case and is equal to the net current divided by the plasma area $\pi b_{p}^{2}$.

For the more realistic case of a high-beta central plasma an additional rotational transform $l_{d}$ is produced by the diamagneric currents in the skin of the helically distorted plasma column. This rotational transform is, for a sharp skin plasma with radius a and for the full torus, 3

$$
z_{d}=\frac{4 \pi B^{2} h R}{(2-B)^{2}}\left\{\frac{B_{l=1}}{B_{z}}\right\}^{2} \frac{1}{(h a)^{2}}\left|\frac{a}{r}\right|^{6} .
$$

This $z_{d}$ can be two orders of magnitude larger than the $:_{\ell}$ given by Eq. (1) but it falls off sharply with $r$. This means that 'd usually does not contribute to the poloidal flux between the outer edge of the plasma and the coil [Eq. (5)]; oniy when a helically shaped quartz tube is used ${ }^{d}$ contributes to that flux early ir. time when the dense plasma is still close to the wall. In that case the net current rises initially faster than given by Eq. (6). When a smooth-bore quartz tube is used, the distortions of the dense plasma and the peak in id develop after the implosion of the dense plasma has taken place.

The large peak in ${ }_{d}$ produces in the conducting plasma just outside the main column an equal and cpposite ${ }^{l} c$ peak to keep the total transform equal to ${ }^{2}$ res (see Fig. 1b). To find the local current densities we differentiate Eq. (10)

$$
\mu_{0} R 2 \pi r j_{z}=\frac{\partial}{\partial r}\left(\imath_{c} r^{2} B_{z}\right) \text {. }
$$

Since the derivative of ${ }^{1} c^{r^{2} B_{z}}$ has opposite signs on either side of the peak, oppositely directed $j_{z}$ currents have to be flowing to generate the $i_{c}$ peak.

A $B=1$ plasma with $a=1 \mathrm{~cm}$ confined in the $\ell_{0,1}$ torus of the first example in Sec. III.B has an ${ }_{d}$ peak of $137 \mathrm{rad}$ and a $z$ current on either side of the peak of $10.9 \mathrm{kA}$. 
It is likely that a diffuse skin will lead to a lowering of the ${ }^{2}$ peak. and the thereby induced $z$ currents. The ibove example shows nevertheless how much the configuration in the experiment can differ from the "standard" configurations where the net $z$ current on any surface is assumed to be zero.

The large localized $j_{z}$ currents are not easily detected in tiie experiment, because they will not affect the net $z$ current. Even oscillations of $\delta_{1}$ a (see Ref. 6) should not show up in the net current because all corresponding local oscillations in $j_{2}$ are screened off by the surrounding low-density plasma region. Note that the description given here uses as its only assumption the presence of a highly conducting, low-density plasma cutside the dense central column. Especially close to the column where most of the effects occur, the presence of this plasma is hardly debatable.

B. Helical $\ell=2$ Field Applied Late in Time

In this second example we apply a relatively weak $\ell=2$ field to as alroady formed pure theta pinch. Neglecting dianagnetic effects of the central plasma we consider the initial state as a low-density, highly conducting plasina in a strong $B_{z}$ field, somehow confined in a torus.

We then apply from the sutside an $\ell=2$ field which in the empty tube would have field components

and

$$
B_{r}=A_{2} r \cos (2 \theta-h z)
$$$$
B_{\theta}=A_{2} r \sin (2 \theta-h z) \text {. }
$$

Image currents in the outer edge of the plasma, at $r=b_{p}$, try to prevent the penetration of thess poloidal fields. The combination of inage currents and poloidal fields pushes the low-density plasma in until its outer surface is is distorted in the same manner as the magnetic surfaces generaced outside the plasma. As a resuit of the distortion of the plasma surface, $\ell=2$ fields are produced inside the plasma, as components of appropriately distorted longitudinal field lines. An important difference with the vacutsm field of Eq. (14) is however, that the rotational transform of the fieis lines in the plasmi must ramain zero. Therefore, a $z$ current in the plasma has to cancel the rotational transform of the $\ell=2$ field. The $l_{\ell}$ from Eq. (2) generates an equal and opposite ${ }^{c}$ which is approximately uniform in space as $l_{\ell}$ is. Thic leads to a uniform current density [from Eq. (10)] 


$$
j_{z}=2 A_{2}^{2} / \mu_{0} h B_{z}
$$

and a corresponding $B_{\theta}$ field, $B_{\theta c}$,

$$
B_{\theta c}=1 / 2 \mu_{o} j_{z} r=\mathrm{rA}_{2}^{2} / \mathrm{hB}_{\mathrm{z}} \text {, }
$$

which turns out to be a factor $A_{2} / h B_{z}$ times the maximun value $A_{2} r$ of the $B_{G}$ camponent of the $\ell=2$ field. Although this factor does not seem to be large in most cases, the component $B_{\theta c}$ obviously destroys the magnetic surfaces, since a rotational transform is necessary to generate the surface in miny revolutions around the torus.

The homogeneous $j_{z}$ of Eq. (15) takes care of the flux $r$ onservation inside the plasma. In addition to this current a net current is needed to compensate for the flux $\phi_{\ell}$ between the coil and the outer edge of the plasma. The difference between the net current [Eqs. (2) and ( $\epsilon$ )] and the volume current [from $E q$. (15)] must flow as a surface current $I_{s}$ at $r=b_{p}$.

$$
I_{s}=\frac{\pi_{A}^{2}}{\pi_{o} h B_{z}}\left\{\frac{b_{c}^{2}-b_{p}^{2}}{\ln b_{c} / b_{p}}-? b_{p}^{2}\right\} .
$$

Figure 1c shows the jumps in ' $c$ ' 'res' and in $E_{\theta c}$ that correspond to this: surface current. As one can see from Eq. (17), Is only disappears when the conducting wall moves in, so that $b_{c}$ approaches $b_{p}$. If one cancels the net current by means of an auxiliary bank, the surface current would change direction but the volume current Eq. (15) would be unaffected. To cancel the volume current also, one could build in a volume current during the formation of the theta pinch so that the volume current would disappear again upon the application of the $\ell=2$ field. In addition to that the net current would have to be canceled. Whereas such a procedure might still be practical when just one auxiliary field is used, it would be impossible co cancel all currents when two or more auxiliary fields are used.

The treatment given in this section resembles the one given by Furth, ${ }^{14}$ but differs in the description of the global flux conservation between the plasma and the coil. 


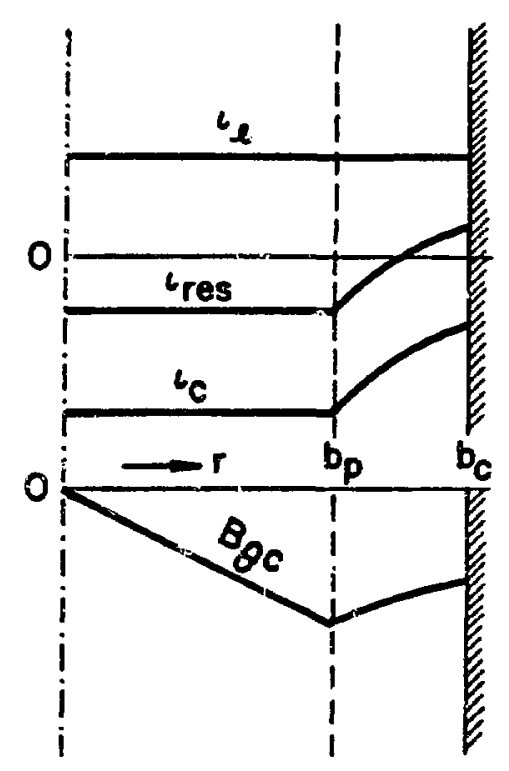

a
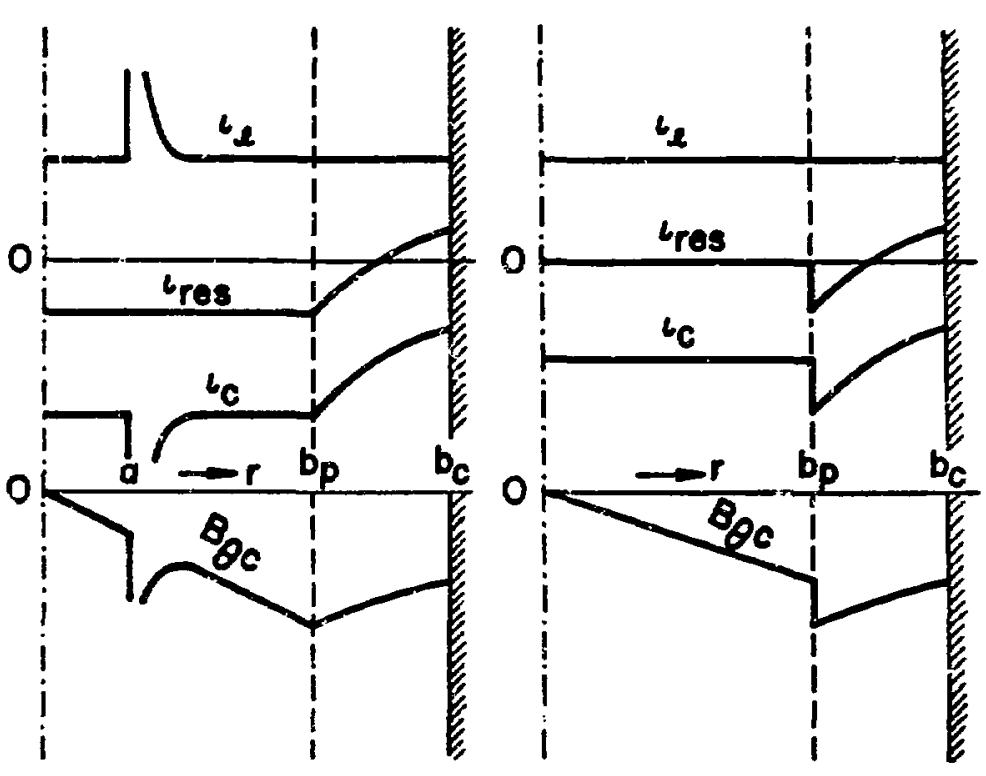

b

C

Fig. 1

Radial distribution of the rotational transforms caused by the helical fields,

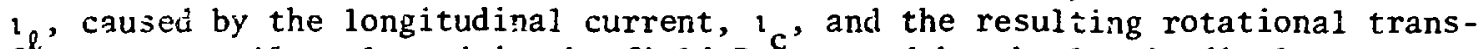
form, ' ${ }^{\prime}$. Also plotted is the field $B{ }^{C}$ caused by the longitudinal curreilt. The outside edge of the conducting plasma is at $b$, the inside of the coil is at $b_{c}$. a) Situation for a low- $B$ Scyllac where the $B^{r}$ field and the $\ell=1$ field are applied simultaneously. Since the flux between $b_{p}^{z}$ and $b$ is kept zero by the plasma, the incoming rotational transform is changed and ${ }^{c}$ a homogeneous $j$ results in the plasma. b) Situation as in a) but with a high- $\beta$ plasma core with ${ }^{2}$ radius a. The helical deformations of the central plasma cause a strong peak in $l_{\text {g }}$ just outside the core. This causes an equal and opposite peak in ? , so that? can stay constant. The i peak corresponds to stroing opposing $z$ currents that do not change the net current. c) An $\ell=2$ field applied to an already fcrmed pure theta pinch. The $\ell=2$ fields distort the central region so that a plasma current has to flow to avoid a change of l $^{2}$. A skin current keeps the flux between $b$ and $b$ zcro. The curves near the axis will be changed somewhat if $a$ high-beta core ${ }^{c}$ is present.

\section{CONCLUSIONS}

- The helical fields in a Scyllac configuration generate a poloidal flux between the outside edge of the plasma and the inside of the coil. The change of this flux during the risetime of the fields induces a net current in the plasma [EG. (i)]. The magnitude of this current is larger for higher \& fields. 
- The net plasma current depends on whether and where a longjeadinal return current can flow in the theta coils. No net current will flow in a ssctor experiment, in a torus one can cance! the net current with an additional bank.

- Volume currents inside the plasma are induced whenever the lucal rotational transform tends to change. These volume currents conserve the fluxes between the magnetic surfaces and hence conserve the rotational transform. This corservation takes place in a wide region, since the low-density plasma between the quartz wall and the central plasma is well conducting on the time scales considered.

- When the helical and the longitudinal field are appiied with the same time history, the volume currents are related to the above-mentioned net current and to the helical displacement of the diamaguetic central plasma.

- When the helical field is applied after the main field, both surfacc and volume currents are needed to conserve the vutside and the inside fluxes.

- Volume currents may flow in a sector experiment, when wall shorting takes place at the ends, or when changes in the rotational transform produce rapid changes in the angular velocities of the plasma.

- Complete cancellation of net current and volume currents in a torus is extremely difficult, especially when more auxiliary fields are used.

- The plasma currents resulting from the flux conservation lead to equilibria which differ from those commonly considered for Scyllac stability calculations.

\section{ACKNOWLEDGMENTS}

The author acknowledges the contributions of Bill Ellis during an early phase of this study and wishes to thank Jeff Freidberg and Werner Riesenfeld for valurable discussions.

\section{REFERENCES}

1. H. Weitzne:, "Free Boundary Long Helical Wavelength Equilibria," Phys. Fluids 14, 658-670 (1971).

2. S. A. Colgate, J. P. Ferguson, H. P. Furth, and R. E. Wright, "A Partly Stabilized Dynamic Pinch," Proc. Second UN Intern. Conf. Peaceful Uses Aî. Energy, Geneva, 1958, Vo1. 32, pp. 140-144. 
3. C. Bobeldijk, R. J. J. Van Heijningen, P. C. T. Van der Laan, L. Th. M. Ornstein, W. Schuurman, and R. F. De Vries, "Properties of a Toroidal Screw yinch Surrounded by a Constant-Pitch Magnetic Ficld," Proc. Third Conf. on Plasma Physics and Contr. Jucl. Fusion Research, Novosibirsk (J968) Vol. 1 , pp. 287-297.

4. J. F. Clarke, "High Beta Flux-Conserving Tokamaks," Oak kidge Report ORNL/ TM1-5429 (1976).

5. K. Sugisaki, "Origin of Axial Current in Scyllac," Los Alamos Scientific Laboratory report LA-6178-MS (December 1975).

6. G. Miller, "Morion of a Plasma Column in a Perturbing Magnetic Field," Phys. Fluids 18, 1704-1709 (1975).

7. E. Fünfer, M. Kaufmann, W. Lotz, J. Neuhauser, G. Schramm, and U. Seiłel, "Linear $\&=1$ Stellarator Experiments Using a Shaped Coil," Report MaxPlanck-Institut für Plasmaphysik IPP $1 / 130(19 ; 3)$.

8. J. L. Johnson, C. R. Oberman, R. M. Kulsrud, and E. A. Frieman, "Some Stable Hydromagnetic Equilibria," Phys. Fluids 1, 281-296 (1958).

9. A. I. Morozov, L. S. Solcv'ev in Reviews of Plasma Physies, Vol. 2, Editor, M. A. Leontovich, (Consultants Bureau, New York, 1966), F. 18 .

10. M. D. Machalek, E. L. Cantre11, W. R. Elilis, and P. C. T. Van der Laan, "I Current Measurements in the Scyllac Torus," Bull. Am. Phys. Soc. $19(1974)^{z}$ 903.

11. P. C. T. Van der Laan, "Currents in the Region Outside Toroidal and Linear Pinches," Proc. 3rd Eur. Sonf. on Contr. Fusion and Plasma Physics, Utrecht (1969), p. 87.

12. I. R. Jones, J. M. Peiry, and F. S. iroyon, "Influence of Limiters on the Penetration of an Oscillating Axial Current in a Straight Pinched Discharge," Nuclear Fusion 14, 497-502 (1974).

13. J. P. Freidberg, Los Mlamos Scientific Laboratory, personal communication, June 1974 .

14. H. P. Furth, "The Stellazator as a Nonlinear Plasma Current Transformer," Phys. Fluids 14, 194-196 (1971). 